\title{
BioéthiqueOnline
}

\section{Art + Bioéthique : quand la recherche en bioéthique quitte les murs de l'université}

\author{
Vincent Couture, Jean-Christophe Bélisle-Pipon et Maude Laliberté
}

Volume 5, 2016

URI : https://id.erudit.org/iderudit/1044274ar

DOI : https://doi.org/10.7202/1044274ar

Aller au sommaire du numéro

Éditeur(s)

BioéthiqueOnline

ISSN

1923-2799 (numérique)

Découvrir la revue

Citer ce document

Couture, V., Bélisle-Pipon, J.-C. \& Laliberté, M. (2016). Art + Bioéthique : quand la recherche en bioéthique quitte les murs de l'université. BioéthiqueOnline, 5. https://doi.org/10.7202/1044274ar
Résumé de l'article

Comment engager le public dans une réflexion sur les enjeux éthiques de notre époque? Guidés par cette question, nous avons développé une plateforme d'échange avec la communauté sur des questions bioéthiques à travers une exposition artistique ainsi qu'une série d'activités de médiation culturelle et scientifique pour les adultes et les enfants. Ce dossier thématique dresse le compte-rendu de ce projet. Art + Bioéthique repose sur une stratégie interdisciplinaire et collaborative qui a amené six jeunes chercheurs en bioéthique à être jumelés à six artistes de la relève afin d'échanger et d'apporter un nouvel éclairage sur une variété d'enjeux éthiques liés à la santé. Ces duos artistes-chercheurs ont chacun développé une oeuvre et un essai sur un thème commun. Notre objectif était de décloisonner l'art et la recherche académique en bioéthique afin de créer des formes hybrides et inédites de diffusion, d'éducation, d'expérimentation et de rencontre. L'expression de la bioéthique à travers l'art constitue une façon innovatrice de transmettre l'aspect sensible de tant de questions éthiques touchant à la santé et au bien-être. À travers ses divers volets, Art+Bioéthique a su rejoindre plusieurs centaines de personnes et les engager dans une réflexion éthique sur des enjeux au coeur de notre société.
Droits d'auteur @ V Couture, J-C Bélisle-Pipon et M Laliberté, 2016

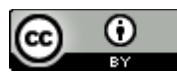

Ce document est protégé par la loi sur le droit d'auteur. L'utilisation des services d'Érudit (y compris la reproduction) est assujettie à sa politique d'utilisation que vous pouvez consulter en ligne. 


\title{
Art + Bioéthique : quand la recherche en bioéthique quitte les murs de l'université
}

\author{
ÉDITORIAL / EDITORIAL \\ Vincent Couture $^{1}$, Jean-Christophe Bélisle-Pipon ${ }^{2,3}$, Maude Laliberté $2-4$
}

Published/publié: 16 Sept 2016

2016 V Couture, J-C Bélisle-Pipon, M Laliberté, Creative Commons Attribution 4.0 International License

\begin{abstract}
Résumé
Comment engager le public dans une réflexion sur les enjeux éthiques de notre époque? Guidés par cette question, nous avons développé une plateforme d'échange avec la communauté sur des questions bioéthiques à travers une exposition artistique ainsi qu'une série d'activités de médiation culturelle et scientifique pour les adultes et les enfants. Ce dossier thématique dresse le compte-rendu de ce projet. Art + Bioéthique repose sur une stratégie interdisciplinaire et collaborative qui a amené six jeunes chercheurs en bioéthique à être jumelés à six artistes de la relève afin d'échanger et d'apporter un nouvel éclairage sur une variété d'enjeux éthiques liés à la santé. Ces duos artistes-chercheurs ont chacun développé une œuvre et un essai sur un thème commun. Notre objectif était de décloisonner l'art et la recherche académique en bioéthique afin de créer des formes hybrides et inédites de diffusion, d'éducation, d'expérimentation et de rencontre. L'expression de la bioéthique à travers l'art constitue une façon innovatrice de transmettre l'aspect sensible de tant de questions éthiques touchant à la santé et au bien-être. À travers ses divers volets, Art+Bioéthique a su rejoindre plusieurs centaines de personnes et les engager dans une réflexion éthique sur des enjeux au cœur de notre société.
\end{abstract}

Mots clés

art, bioéthique, exposition, recherche, transfert des connaissances, interdisciplinarité

\section{Summary}

How should one engage the public in a reflection on the ethical issues of our time? Guided by this question, we developed a platform for exchange with the community on bioethical issues via an art exhibition and a series of cultural and scientific mediation activities for adults and children. This thematic dossier presents the report of the project. Art + Bioethics is based on an interdisciplinary and collaborative strategy that paired six young bioethics researchers with six emerging artists to share and shed new light on a variety of ethical issues related to health. These artist-researcher duos each developed a work and an essay on a common theme. Our goal was to break down barriers between art and academic research in bioethics and to create hybrids and new forms of dissemination, education, experimentation and meeting. The expression of bioethics through art represents an innovative way to transmit the sensitive aspect of so many ethical issues of health and well-being. Through its various components, Art + Bioethics was able to join several hundred people and engage in ethical reflection on issues that are at the heart of our society.

\section{Keywords}

art, bioethics, exposition, research, knowledge transfer, interdisciplinarity 
Affiliations des auteurs / Author Affiliations

${ }^{1}$ Programmes de sciences cliniques, Université de Sherbrooke, Sherbrooke, Canada

${ }^{2}$ Programmes de bioéthique, Université de Montréal, Montréal, Canada

${ }^{3}$ Institut de recherche en santé publique de l'Université de Montréal (IRSPUM), Montréal, Canada

${ }^{4}$ Centre de recherche interdisciplinaire en réadaptation du Montréal métropolitain (CRIR), Montréal, Canada

\section{Correspondance / Correspondence}

Vincent Couture, vincent.couture@usherbrooke.ca

\section{Remerciements}

Nous souhaitons remercier très chaleureusement la Caisse Desjardins de Lorimier-Villeray, le Centre de recherche en éthique (CRÉ) et le festival Nuit blanche (Montréal) pour leurs contributions financières. Nous voudrions souligner l'apport inestimable de la revue BioéthiqueOnline, de son comité éditorial pour son soutien ainsi qu'à tous les éditeurs qui ont généreusement participé à la révision de ce dossier. Ce projet a bénéficié de l'apport inestimable de nombreux bénévoles qui ont apporté leur aide lors des différents évènements, dont plus spécifiquement Nathalie Voarino lors de la Nuit blanche et France Geoffroy pour sa présence lumineuse lors de sa participation à la performance de l'artiste Maël Le Mée. Un immense merci à Liliane Audet, Martine Vinuesa, Mathieu Vinuesa, Mariane Stratis et Marion Paquette pour leur aide lors des ateliers de médiation ainsi qu'au photographe Alexandre Campeau-Vallée pour sa contribution au catalogue. Tout ce projet n'aurait pu se faire sans la participation des membres de nos binômes : Jonas-Sébastien Beaudry, Annie Carrier, Stephanie Coleman, Victoria Doudenkova, Audrey Kinkead, Arkadi Lavoie-Lachapelle, Mael Le Mée, Jean-Frédéric Ménard, Mathieu Noury, Dominick RathwellDeault, Grace Stokes et Karine Turcot.

Les auteurs tiennent à remercier le concours Forces Avenir pour la reconnaissance octroyé au projet. Plus spécifiquement, VC remercie le Fonds de recherche du Québec-Santé (FRQ-S), le Fonds de recherche du Québec-Société et culture (FRQ-SC), le Réseau de formation en recherche périnatale du Québec IRSC (QTNPR), la Fondation Desjardins, le CRÉ et le Centre de recherche du Centre hospitalier de l'Université de Sherbrooke (CRCHUS) pour leur soutien. JCBP voudrait remercier le soutien financier du FRQ-S et de l'Unité SOUTIEN-SRAP. ML, pour sa part, souhaite remercier le soutien financier du FRQ-S.

\section{Conflit d'intérêts}

VC est éditeur exécutif de la revue BioéthiqueOnline et éditeur de la section Inspirée par la bioéthique. JCBP est co-fondateur de la revue et a été jusqu'à tout récemment éditeur exécutif. ML a été membre du comité éditorial et éditrice de section (Études de cas, Inspiré par la bioéthique).

\section{Acknowledgements}

We wish to thank the Caisse Desjardins de LorimierVilleray, the Centre for Ethics Research (CRÉ) and the Nuit Blanche festival (Montreal) for their financial contributions. We would like to highlight the invaluable contribution of the journal BioéthiqueOnline, its editorial board for their support and all the editors who generously participated in the review of this dossier. This project benefited from the invaluable contribution of many volunteers who provided assistance at various events, including specifically Nathalie Voarino during Nuit Blanche and France Geoffroy for her luminous presence during his participation in the performance of artist Maël Le Mée. A huge thanks to Liliane Audet, Martine Vinuesa, Mathieu Vinuesa, Mariane Stratis and Marion Paquette for their help in the mediation workshops as well as photographer Alexandre CampeauVallée for his contribution to the catalog. The whole project would not have been possible without the participation of the duo members: Jonas-Sébastien Beaudry, Annie Carrier, Stephanie Coleman, Victoria Doudenkova, Audrey Kinkead, Arkadi Lavoie-Lachapelle, Mael Le Mée, JeanFrédéric Ménard, Mathieu Noury, Dominick RathwellDeault, Grace Stokes and Karine Turcot.

The authors wish to thank the Forces Avenir contest for the recognition granted to the project. More specifically, VC thanks the Quebec Research Fund-Health (FRQ-S), the Quebec Research Fund-Society and Culture (FRQ-SC), the Québec Training Network in Perinatal Medicine (QTNPR), the Fondation Desjardins, the CRÉ and the Research Centre of the University of Sherbrooke Hospital (CRCHUS) for their support. JCBP would like to thank the financial support of the FRQ-S and the SUPPORT-SRAP Unit. ML, for her part, wishes to acknowledge the financial support of the FRQ-S.

\section{Conflicts of Interest}

VC is currently Executive Editor and interim Section Editor for Inspired by Bioethics of BioéthiqueOnline. JCBP is cofounder of the journal and was until recently an Executive Editor. ML was on the editorial board and Section editor (Case Studies, Inspired by Bioethics).

\section{Introduction}

Du 25 février 2016 au 21 mars 2016, la galerie Espace Projet à Montréal a abrité l'exposition Art + Bioéthique présentant le travail conjoint d'artistes et de bioéthiciens. Cet évènement inusité mélangeant l'art et la bioéthique n'était en fait que la partie la plus visible d'un projet regroupant une série d'activités de médiation autant scientifique que culturelle et culminant avec la publication de ce 
dossier thématique de BioéthiqueOnline. Ce projet, que nous avons démarré au printemps 2015 (avec l'aide des commissaires Catherine Barnabé et Marianne Cloutier assistées d'Aïda Lorrain) avait pour but de développer un carrefour entre l'art, la recherche et le public afin de sensibiliser, d'apprendre et de stimuler des débats publics sur des enjeux éthiques liés à la santé et au bien-être des individus. Pour réaliser cette intersection, nous avons constitué six binômes composés d'un jeune chercheur en bioéthique et d'un artiste professionnel de la relève afin de faire émerger de cette rencontre un travail commun, soit un essai pour le bioéthicien et une œuvre pour l'artiste.

Alors que la bioéthique occupe une place grandissante dans nos sociétés pluralistes et démocratiques par son questionnement sur les grands enjeux éthiques liés à la santé et au développement des biotechnologies, paradoxalement les chercheurs rencontrent plusieurs difficultés dans le transfert de leurs connaissances et un fossé persiste entre la recherche universitaire en éthique et le grand public [1]. Ce manque d'intégration, entre autres identifié par Désy et collaborateurs, appelle à explorer des nouveaux modes de transfert des connaissances [1]. En lien avec la visée sociale de la bioéthique, l'objectif ultime de cette démarche est de faire bénéficier à la collectivité des savoirs développés par la recherche [2]. De tous les véhicules pouvant être utilisés pour ce transfert [1], l'art représente un de ces mécanismes recevant une attention croissante comme en témoigne la reconnaissance par la revue britannique Lancet Oncology du projet réalisé conjointement par la chercheure Astrid Brousselle et l'artiste Anouk Sugàr [3]. Pour diffuser les résultats des recherches de Brousselle sur le cancer, Sugàr a illustré les résultats par collages reproduits sur des cartes postales distribuées au métro Longueuil (Québec) durant l'heure de pointe [3]. Plus spécifiquement dans le champ de la bioéthique, l'introduction d'une série de conférences sur le thème de l'art et de la bioéthique ainsi qu'une exposition figurant au programme du congrès mondial de 2016 de l'International Association of Bioethics représentent un autre exemple de cet intérêt.

Cette rencontre de l'art et de la bioéthique sous l'angle d'un apport extra-disciplinaire pose toutefois un défi, à la fois, théorique et pratique. En effet, est-ce que l'art et la bioéthique sont compatibles? Comment intégrer ces deux domaines? Dans la prochaine section, nous analyserons la contribution mutuelle de l'art et de la bioéthique. Ceci nous amènera à préciser l'apport d'Art + Bioéthique et à mettre en lumière la nature de l'échange qui s'est opéré entre les participants au projet et le grand public. Ensuite, nous présenterons rapidement les textes formant ce dossier thématique. Ceux-ci se composent des essais des bioéthiciens ainsi que d'un appareil critique et descriptif développé par les commissaires de l'exposition : Marianne Cloutier, Catherine Barnabé et Aïda Lorrain. Finalement, nous présenterons l'ensemble des collaborateurs au projet.

\section{Quel apport mutuel pour l'art et la bioéthique?}

La bioéthique et l'art ont plusieurs points en commun dont les principaux sont de toucher aux émotions, de référer au sensible et, pour utiliser un langage philosophique, de fonctionner par jugement de valeur pour déterminer ce qui est beau, bien, de bon goût ou souhaitable. De plus, pour paraphraser Tsitas, la bioéthique traite de la vie, de la mort, de la souffrance et de l'existence humaine, ce qui est également à la base du travail artistique [4]. Bien que ce chevauchement soit reconnu, la nature de l'apport d'une discipline à l'autre est encore débattue [5]. L'histoire récente de la bioéthique a vu plusieurs tentatives de brouillage de ces genres dont les plus connus sont l'éthique narrative [6], le tournant personnel en bioéthique [7], l'éthique visuelle [8], le bio-art [9], le sci-art [10] et la recherche en santé fondée sur l'art [11]. Ces approches reposent sur l'idée que l'art et l'éthique sont deux grands discours nous permettant de comprendre notre époque contemporaine et que leur combinaisation offre l'opportunité de bénéficier des outils de l'autre discipline. En analysant plus attentivement la littérature à l'intersection de l'art et de la bioéthique, il est possible d'identifier quatre apports - souvent mutuels - de l'art et de la bioéthique qui seront développés dans les paragraphes qui suivent: (a) l'art comme commentaire bioéthique, (b) l'art et la bioéthique comme 
complémentaires, (c) la combinaison de l'art et de la bioéthique pour développer la réflexivité et (d) la rencontre de l'art et de la bioéthique pour l'ouverture d'un débat public.

\section{L'art comme commentaire bioéthique}

L'apport le plus souvent cité dans la littérature repose sur l'idée que l'art révèle alors que la bioéthique explique. Cette approche est bien incarnée par ce que les anglophones nomment les « medical humanities ». À l'origine de cette discipline repose l'idée que l'enseignement de la culture et des sciences humaines peuvent améliorer la formation et la sensibilité des étudiants en santé. Dans ce cursus, la bioéthique s'inspire de l'art. Par exemple, à partir d'une toile de Francisco Goya, un professeur invite ses étudiants à analyser la narration de l'œuvre. Au fil des discussions, le groupe développe une réflexion sur la relation médecin-patient, la profession médicale, le rapport au soin et la disparition du soin pour une offre désincarnée de traitement [12].

Ce type d'apport ne se limite pas aux medical humanities et se retrouve aussi dans la posture critique de la "visual bioethics». Cette branche récente de la bioéthique défend l'idée que les images véhiculent une rhétorique qui influence, à sa façon, la réflexion normative [8]. Ce champ développe une analyse des images plus proche de l'art et de la communication pour décortiquer les images qui tapissent le débat bioéthique (par exemple, les images utilisées par les groupes pro-vie dans le débat américain sur l'avortement) [8]. Cette approche naît d'une critique du logocentrisme propre à la bioéthique et soutient qu'il faut s'ouvrir à la sensibilité et à l'affect véhiculés par les images.

L'image en mouvement (cinéma, documentaire, séries télés) ou la littérature sont aussi régulièrement citées comme sources d'inspiration ou de critique pour la bioéthique. Les narrations visuelles ou textuelles peuvent ainsi être utilisées comme façons sensibles pour introduire des dilemmes sociaux et culturels ou tout simplement raconter la bioéthique [10]. Elles peuvent aussi servir comme étude de cas, de concepts ou de théories. Cette utilisation transcende les médiums pour inclure des genres et les multiples déclinaisons de la culture populaire [13]. La science-fiction avec ses Frankenstein, Gattaca et Meilleur des mondes occupe une place paradigmatique en offrant la possibilité d'anticiper des problématiques éthiques futures en disant " et si c'était comme ça? " tout en suggérant très souvent de procéder avec prudence [4].

Un autre exemple abondamment discuté en bioéthique est celui du bio-art [5], cette pratique artistique qui utilise des tissus vivants, des bactéries, des organismes vivants et, de façon large, les processus et les caractéristiques du vivant. À la manière de la science-fiction, ces œuvres nous permettent d'imaginer un futur proche façonné par les biotechnologies ainsi que ses implications éthiques. Par exemple, Genetic Trace de l'artiste Susana Soares propose d'améliorer notre compréhension du monde par l'ajout de nouveaux organes semblables à de longs cils situés au bout des ongles et des sourcils [14]. Un autre exemple est celui de l'artiste Steve Kurtz qui, se nourrissant de la peur du bioterrorisme, a créé de nouveaux organismes vivants afin questionner la dérive sécuritariste des sociétés occidentales [15]. Un dernier exemple est le travail ni vraiment artistique ni scientifique de l'anatomiste Günter von Hagens. Selon Yechiel Michael Barilan, l'exposition Bodyworld de von Hagens ouvrirait un espace de réflexion sur nos devoirs envers les morts en offrant aux visiteurs un certain éthos de la récupération cadavérique [16].

\section{L'art et la bioéthique comme complémentaires}

Un premier apport de l'art comme commentaire bioéthique peut être vu de façon unidirectionnelle : l'art contribue à appuyer (voire illustrer) le discours de la bioéthique. Par contre, un second apport, moins développé dans la littérature, montre une complémentarité entre les deux domaines. Vision plus circulaire de l'art et de la bioéthique, celle-ci soutient que, par la rencontre, peuvent émerger de nouvelles possibilités de sens [10]. Par exemple dans son analyse du bio-art, Stephen Webster soutient que le discours bioéthique a tous les outils pour bien expliquer la transformation de la vie 
humaine, mais c'est l'art qui analyse le mieux la déstabilisation de nos attitudes envers un monde naturel évoluant avec une inquiétante étrangeté [10]. L'art vient ainsi compléter la bioéthique et ne se limite pas à incarner l'objet d'un simple commentaire. L'art permet de remettre en question les catégories tout en développant une vision fluide du monde de façon créative et prospective [5]. Inversement, la bioéthique peut servir à donner un contenu éthique à l'art et à l'amener à prendre position [5,7].

\section{La combinaison de l'art et de la bioéthique pour développer la réflexivité}

La rencontre de l'art et de la bioéthique ouvre la possibilité d'un troisième type d'apport lié au développement d'une réflexivité. Par exemple, le projet multidisciplinaire InVisible Difference en permettant la rencontre de danseurs handicapés, de chorégraphes et de spécialistes du droit a permis de réfléchir sur les conceptions médicales du corps et comment une approche bioéthique pourrait s'en détacher [17]. Ceci n'est pas sans faire penser au travail du chorégraphe français Jérôme Bel et de l'artiste québécoise France Geoffroy. En poussant plus loin la réflexion de Chambers sur ce point [7], nous considérons que cette réflexivité émerge mutuellement autant chez l'artiste que le bioéthicien. Pour Chambers, à la manière des « ready made » de Marcel Duchamp, tel son célèbre urinoir exposé en 1917 à New York, le bio-art nous invite à repenser la nature des objets. En refaçonnant les outils de la génétique, le bio-art amène le spectateur à considérer comment la science - et par extension la bioéthique - acquiert son autorité et développe sa propre rhétorique de ses objets [7]. Ainsi, en déplaçant un objet du monde de l'art vers celui de la bioéthique (ou inversement), cet objet acquiert de nouvelles significations qui mettent en lumière la logique de fonctionnement de cet objet. Autant l'art peut amener la bioéthique sur sa position d'énonciation (autant argumentative que corporel comme dans le cas des disability studies [7]), la bioéthique amène l'artiste à se questionner sur les jugements et les valeurs tenues pour a priori dans son œuvre. Cette réflexivité, naissant de la rencontre de ces disciplines, permet donc de remettre en question ce qui est tenu pour acquis autant en art qu'en bioéthique [5]. Ceci ouvre la possibilité d'un dépassement disciplinaire en développant un regard plus juste, pertinent, critique, et esthétique du monde.

\section{La rencontre de l'art et de la bioéthique pour l'ouverture d'un débat public}

Un dernier apport réfère à la possibilité d'ouvrir un espace d'échange et de dialogue tout au long des frontières mouvantes entre ces deux domaines [5]. L'art a cette capacité puissante comme médium de présenter des enjeux éthiques au grand public [7]. Par exemple, l'art peut médiatiser les nouvelles technologies en les rendant plus accessibles à un plus grand public [18]. En prenant une voie autre que celle du discours de l'expert, l'art permet de représenter ces développements par des interventions éclairantes, avec toutefois quelques incertitudes et ambivalences $[5,18]$. Le travail de l'art permet aussi de présenter dans un tout des questions complexes qui, par l'entremise du discours bioéthique, prendrait une forme moins accessible. Comme le soutien Stacey, l'art ouvre la science à des audiences différant des congrès scientifiques [18]. Cet apport est d'autant plus utile pour la bioéthique que celle-ci revendique un rôle de médiation sociale sur les grands enjeux éthiques. Inversement, la bioéthique peut, à son tour, mettre le doigt sur des questions interpellant directement la population, rendant ainsi plus concrètes les voies de dialogues entre l'art et le social. Cette capacité de la bioéthique de donner une voix au sensible demeure toutefois, au sein de la littérature, un sujet à développer.

Ces quatre types d'apports montrent que la discussion et le métissage entre l'art et la bioéthique sont déjà enclenchés. Cependant, ce type de projet n'implique encore que très peu la communauté académique, et encore plus rarement la communauté bioéthique. Jusqu'à présent, la tendance forte demeure l'utilisation unidirectionnelle de l'art par la bioéthique. C'est dans cet espace interstitiel et dans l'idée d'opérer un véritable dialogue que s'est développé le projet Art + Bioéthique. Notre but était de voir comment ces deux domaines peuvent se compléter, s'inspirer, se réfléchir, se dépasser, rayonner et s'ouvrir à un nouveau champ de pratiques résonnant et interpellant le public. 


\section{Retour sur le dispositif à la base d'Art + Bioéthique}

Contre une approche unidirectionnelle allant de la bioéthique au public en passant par l'art comme médium, nous avons voulu développer une approche bidirectionnelle, voire multidirectionnelle, permettant des interactions personnelles et continues entre les différents acteurs $[1,19]$. C'est-à-dire qu'à l'approche de l'art comme commentaire ou illustration, nous avons opté pour une approche complémentaire. Nous avons recherché cette complémentarité et tablée à établir des jalons permettant son expression, notamment à travers l'idée de binôme artiste-chercheur, comme moyen pour créer une relation égalitaire et horizontale. Nous recherchions aussi à offrir une occasion aux participants de s'ouvrir à un tout nouvel univers et à développer une expérience unique qui les suivront tout au long de leur carrière, permettant ainsi d'influer sur l'évolution de leur domaine respectif et faciliter les rencontres et les rapprochements futurs entre le rationnel et le sensible.

À cause du caractère novateur de cette proposition, on peut dire qu'Art + Bioéthique incarne un laboratoire éphémère en transfert des connaissances. À partir de ce laboratoire, nous avons réalisé plusieurs expériences d'hybridation dont les résultats avaient pour but ultime de partager à un public large des travaux artistiques et bioéthiques pour susciter le débat et la réflexion. II nous est possible de "mesurer" la réalisation de cet objectif tant de manière quantitative, plusieurs centaines de personnes ont assisté et participé aux différentes activités, que de façon qualitative, par la richesse, la profondeur et la pertinence des échanges que nous et nos binômes avons pu avoir entre nous et avec les visiteurs de l'exposition.

II nous faut reconnaître, cependant, que l'objectif de transfert des connaissances, nous amène à un résultat beaucoup plus mitigé. D'un côté, on peut affirmer qu'en raison de la popularité des activités, nous avons pu sensibiliser un public hybride, ayant des affinités soit plus artistiques ou bioéthiques, aux pratiques de l'autre domaine. En ce sens, l'ouverture d'un espace à un public large a pu être observée. Le mélange des problématiques et des démarches artistiques a aussi offert une vision plus large et réflexive de la bioéthique et de l'art aux spécialistes souvent cloisonnés dans des approches restreintes. Dans une entrevue sur Art + Bioéthique, la bioéthicienne Victoria Doudenkova disait justement que « (I)a bioéthique est un domaine qui touche des cordes sensibles chez les gens. Elle appartient à tout le monde, pas seulement à la science. L'art permet justement d'aller chercher ces aspects sensibles que les articles scientifiques ne peuvent pas exprimer » [20]. Lors de son artist talk, Stephanie Coleman, à propos de son projet Na-no-body, nous a exprimé l'influence de cette œuvre sur sa démarche artistique globale [21]. Coleman nous a, entre autres, entretenu de sa collaboration avec Mathieu Noury. L'aspect « low-tech » de son œuvre contraste radicalement avec les travaux de Noury touchant aux technologies de pointe développées par la nano-médecine et amène à réfléchir sur ce que masque les artifices de la technique [22]. Un autre exemple est l'évolution du travail conjoint de l'artiste Mael Le Mée et du bioéthicien et juriste Jonas-Sébastien Beaudry. Dans son travail sur l'hybridation des corps et la « fabrique contemporaine des corps », Mael Le Mée [23] cherche à explorer les limites du monde et de la morale, objectif qui peut facilement entrer en conflit avec le travail de la bioéthique portée, pour plusieurs, vers la quête d'une justice sociale consensuelle. Dans la rencontre de Le Mée et Beaudry, cet écart initial s'est résorbé par l'exploration par Beaudry de nouveaux outils provenant de la théorie critique et de la psychanalyse afin de donner sens à cette expérience limite [24].

D'un autre côté, il semble y avoir eu un excédent de sens qui n'a pu être assimilé autant par les artistes, les bioéthiciens que le grand public. Chaque œuvre, chaque essai et chaque rencontre nous est apparu avoir une part d'incommensurabilité. Pour le dire autrement, la véritable rencontre (entre l'art et la bioéthique, entre les connaissances à transférer et leurs destinataires, entre le rationnel et le sensible) ne s'est pas opérée tout le temps et pour tous. Quelques fois nous avons vu apparaître un fossé entre nos deux univers soit en entendant les bioéthiciens demander plus de discours et d'explications, alors que les artistes cherchaient plus de liberté et de sensibilité, ou soit face à un public parfois dubitatif. Peut-être que considérer cette rencontre sous l'angle d'un transfert de 
connaissances empêche de voir le transfert d'affects presque imperceptible qui s'est opéré entre des individus provenant d'univers si différents?

En optant pour la diversité des tactiques - en référence à la discussion ayant suivi la conférence d'Annie Carrier - une des conséquences est que nous avons plutôt vu émerger une expérience vivante plutôt qu'un mécanisme de transfert des connaissances. Certaines rencontres ont mené à de nouveaux projets. Par exemple, Jean-Frédéric Ménard et Arkadi Lavoie-Lachapelle, suite à leur rencontre, ont développé un contrat pour la vente d'œuvre d'art avec un droit de suite offrant à l'artiste la chance de bénéficier financièrement de la revente de ses œuvres. Au sein de l'ensemble des binômes, leurs rencontres ont également mené à une meilleure compréhension mutuelle de la manière dont chacun des domaines performe le monde. Ceci suggère que la rencontre entre l'art, la bioéthique et le public ne peut être qu'éphémère et se réaliser sous forme d'épiphanies qui n'ont rien à voir avec le langage, trop souvent, austère de la connaissance et plus avec celui du sensible qui unit et permet de rejoindre les arts, la bioéthique et le grand public.

\section{Présentation des textes formant le dossier thématique}

Ce dossier thématique retrace les principaux moments de cette grande aventure : de la genèse du projet à la publication de ce dossier. II est constitué des essais produits par les bioéthiciens et de leurs réflexions inspirées tant dans le style, dans la méthode que dans le propos par leur rencontre des artistes. Suivant chacun des essais, nous avons ajouté une reproduction des œuvres développées par les artistes en lien avec la démarche des bioéthiciens avec qui ils étaient associés. La dernière partie du dossier offre la perspective des commissaires sur la dimension et la qualité artistique du projet. Ainsi, les textes des commissaires de l'exposition Marianne Cloutier et Catherine Barnabé analysent la contribution artistique de chaque œuvre sous l'angle du travail des bioéthiciens. Le dossier se termine par le compte-rendu rédigé par Aïda Lorrain et décrivant l'ensemble des activités de médiations scientifiques et culturelles qui ont constitué Art + Bioéthique.

\section{Ceux et celles qui ont rendu possible le projet et quil l'ont fait vivre}

Puisqu'à la base même du projet, il y avait rencontre, il est nécessaire de souligner la participation et la contribution de ceux et celles qui ont rendu Art + Bioéthique possible.

\section{Artistes}

Stephanie Coleman (Canada) - Stephanie applique le langage propre au textile à divers médias tel que les objets trouvés, la peinture, le dessin et le travail du bois. Elle s'intéresse à l'histoire et à la biologie comme disciplines aux multiples facettes qui permettent sans cesse la découverte de nouveaux éléments.

Audrey Kinkead (Canada) - Audrey observe le vivant, étudie les phénomènes visibles et invisibles qui se manifestent autour d'elle. Ses projets sont pensés comme des laboratoires de gestes ténus et poétiques questionnant les relations que l'humain entretient avec l'animal. Elle veut repenser les interstices, les réseaux tissés entre espèces, en ignorant les conventions hiérarchiques et souhaite aborder le rapport au vivant avec une pincée d'humour et une vision moins anthropocentriste.

Arkadi Lavoie-Lachapelle (Canada) - Arkadi par des manœuvres, des installations en galerie ou des performances, crée des situations qui questionnent les idéologies productiviste et individualiste ambiantes et y opposent une certaine résistance.

Mael Le Mée (France) - Mael développe une pratique artiste transdisciplinaire (performance, installation, texte, audiovisuel, théâtre, internet, etc.) qui va de brancher des légumes vivants sur des ordinateurs, à poser des bombes dans des festivals, en passant par raconter des histoires de 
fantômes aux enfants, donner des conférences très sérieuses sur des sujets qui n'existent pas encore, installer des machines à remontrer le temps, ou vendre des organes de confort.

Grace Stokes (Royaume-Uni) - Grace travaille sur la représentation des frontières poreuses entre art et science. Elle s'intéresse aux relations entre ces deux contextes culturels et à comment l'art a la possibilité de représenter à la fois l'esthétisme et l'idéologie des développements technologiques.

Karine Turcot (Canada) - Karine crée à partir de diverses disciplines telles que la sérigraphie, l'installation, la sculpture, la photographie, l'animation, le livre, le dessin, la céramique, la scénographie, et la performance. Elle s'intéresse à la manière dont nous définissons les catégories de perception que nous attribuons aux choses qui nous entourent.

\section{Bioéthiciens}

Jonas-Sébastien Beaudry (University of British Columbia) - Jonas-Sébastien effectue ses recherches sur l'égalité et inclusion sociale des personnes handicapées. Il est docteur et professeur adjoint à la Allard School of Law, UBC, Vancouver.

Annie Carrier (Université de Montréal) - Annie est ergothérapeute et stagiaire postdoctorale à la Chaire de recherche Connaissances, Politiques et Santé affiliée à la Faculté des sciences infirmières et à l'Institut de Recherche en Santé publique de l'Université de Montréal. Dans son stage postdoctoral, elle s'intéresse aux stratégies de transfert de connaissances auprès des décideurs organisationnels et politiques et aux aspects éthiques qui y sont rattachés.

Victoria Doudenkova (Université de Montréal) - Victoria est étudiante au doctorat en sciences biomédicales (option bioéthique) à l'Université de Montréal. Ses recherches portent sur la mise en évidence des enjeux qui entourent le soin du syndrome des ovaires polykystiques.

Jean-Frédéric Ménard (University College London) - Jean Frédéric effectue ses recherches sur l'exploration et l'amélioration des normes dans la relation de soin en néonatalogie. Il est doctorant en droit à l'University College London.

Mathieu Noury (Université d'Ottawa) - Mathieu s'intéresse aux enjeux éthiques de l'application clinique de la nanomédecine et de la médecine régénératrice. Il est chercheur post-doctorant à l'École d'études sociologiques et anthropologiques de l'Université d'Ottawa.

Dominick Rathwell-Deault (Université de Montréal) - Dominick s'intéresse au concept de responsabilité morale des médecins vétérinaires en regard de leurs patients animaux. Elle est doctorante en sciences cliniques à l'Université de Montréal à St-Hyacinthe.

\section{Organisateurs}

Catherine Barnabé (Espace Projet) - Catherine est commissaire et auteure indépendante, en plus d'être co-fondatrice d'Espace Projet pour lequel elle assure la co-direction depuis 2012. Elle est détentrice d'une maîtrise en études des arts de l'Université du Québec à Montréal (2011).

Jean-Christophe Bélisle-Pipon (Université de Montréal) - Jean-Christophe est candidat au doctorat en Sciences biomédicales (option bioéthique). Ses recherches portent principalement sur la responsabilité des acteurs impliqués dans des activités commerciales et promotionnelles ciblant directement les patients. Alors que sa formation de premier cycle en physique le menait vers les sciences pures, son intérêt pour le domaine de la santé et la bioéthique en général s'est développé au fil de son expérience au sein de l'industrie biopharmaceutique. Pour occuper ses temps libres, Jean-Christophe se passionne pour la gouvernance de la recherche, sa pertinence sociale et son rayonnement. 
Marianne Cloutier (Université de Montréal) - Marianne poursuit actuellement un post-doctorat au département de sciences biologique de l'Université de Montréal. Dans le cadre de ses recherches, elle s'intéresse aux questions éthiques, politiques, sociales et philosophiques qui émergent de l'intégration du vivant en art et du détournement des outils, des techniques et des savoir-faire scientifiques par l'artiste. Son projet de recherche en cours, La fabrique du bioart, se penche plus particulièrement sur la spécificité des processus créatifs de cette forme d'art. À titre de chargée de cours, elle a enseigné l'histoire de l'art au collégial et dans diverses université (UQAM, Université d'Ottawa, Université de Montréal [à partir de septembre 2016]).

Vincent Couture (Université de Sherbrooke) - Vincent est candidat au doctorat aux Programmes de sciences cliniques de l'Université de Sherbrooke. Sa thèse porte sur les services reproductifs transfrontaliers. À partir de recherches ethnographiques dans des cliniques canadiennes de fertilité, il développe une réflexion bioéthique sur la situation canadienne de ce type de déplacements médicaux. II travaille au Laboratoire transdisciplinaire de génétique, médecines et sciences sociales de la Pre Chantal Bouffard.

Maude Laliberté (Université de Montréal) - Maude est candidate au doctorat en Sciences biomédicales (option bioéthique) à l'Université de Montréal. Elle est physiothérapeute et détentrice d'une maîtrise en sciences biomédicales option réadaptation et d'une mineure en éthique et droit. Elle est également professeure adjointe de clinique à l'École de réadaptation de l'Université de Montréal où elle enseigne l'éthique professionnelle. Ses travaux analysent les facteurs influençant l'allocation des ressources.

Aïda Lorrain (Espace Projet) - Aïda est une artiste et commissaire indépendante vivant à Montréal, d'origine québécoise et iranienne. Elle détient un baccalauréat en arts visuels et médiatiques de l'Université du Québec à Montréal. À travers une pratique artistique protéiforme, elle s'intéresse aux espaces hybrides créés par le langage poétique, la fragmentation, les anachronismes, les flux d'échange et le mouvement.

\section{Références}

1. Désy M, Hughes D, Filiatrault F. Des pistes de solution pour une meilleure intégration des considérations éthiques en santé publique. Revue cananadienne de santé publique. 2014;105(2):e142-e5.

2. Université de Montréal. Le transfert de connaissances.

3. Lobo P. Collages for cancer. Lancet Oncology. 2016;17(2):149.

4. Tsitas E. The role of the creative arts in bioethical debates. Queensland University of Technology Law \& Justice Journal. 2006;6(2):255-65.

5. Macneill $P$, Ferran $B$. Art and bioethics: shifts in understanding across genres. Journal of Bioethical Inquiry. 2011;8(1):71-85.

6. Charon R. Commentary on 'Creative expressive encounters in health ethics education: Teaching ethics as relational engagement.' Teaching and Learning in Medicine. 2009;21(2):163-5.

7. Chambers T. The art of bioethics. Hastings Center Report. 2005;35(2):3.

8. Lauritzen P. Visual bioethics. American Journal of Bioethics. 2008;8(12):50-6.

9. Kac E, editor. Signs of Life. Cambridge (Mass): MIT Press; 2007.

10. Webster S. Art, science and the public. In: Turney J, éditeur. Engaging Science: Thoughts, deeds, analysis and action. Londres GB: Wellcome Trust; 2006. p. 74-9.

11. Lafrenière D, Hurlimann T, Menuz V, Godard B. Health research: ethics and the use of artsbased methods in knowledge translation processes. International Journal of the Creative Arts in Interdisciplinary Practice. 2012(11):1-26.

12. Arawi TA. Medicine and the arts. Self-portrait with Dr. Arrieta by Francisco José de Goya y Lucientes. Commentary. Academic Medicine: Journal Of The Association Of American Medical Colleges. 2011;86(1):114-5. 
13. Behrmann J. For the creative spirits out there: bioéthiqueonline inaugurates a publication venue for creative and artistic works in bioethics / Pour les esprits créatifs émergents:

BioéthiqueOnline inaugure une section spéciale pour les travaux créatifs et artistiques en matière de bioéthique. BioéthiqueOnline. 2013;2/Ed2

14. Soares S. Genetic trace.

15. Annas GJ. Bioterror and 'bioart' - A plague o' both your houses. The New England Journal of Medicine. 2006;354(25):2715-20.

16. Barilan YM. Bodyworlds and the ethics of using human remains: a preliminary discussion. Bioethics. 2006;20(5):233-47.

17. Harmon SHE. The invisibility of disability: using dance to shake from bioethics the idea of 'broken bodies'. Bioethics. 2015;29(7):488-98.

18. Stracey F. Bio-art: The ethics behind the aesthetics. Nature Reviews Molecular Cell Biology. 2009;10(7):496-500.

19. Macneill P. Ethics and the arts. Dordrecht: Springer; 2014. 273 p.

20. Andreani E. Art + Bioéthique, un mariage surprenant. Quartier Libre. 2016.

21. Coleman S. Na-no-body. BioéthiqueOnline. 2016;5/19.

22. Noury M. Na-no-body: De l'oubli du corps sensible en nanomédecine. BioéthiqueOnline. 2016;5/20.

23. Le Mée M. Mael-e(s)t-France. BioéthiqueOnline. 2016;5/27.

24. Beaudry J-S. The anxious heart of injustice: negative affective responses to disabilities. BioéthiqueOnline. 2016;5/28.

\section{Liens vers les contributions au dossier thématique}

- Lavoie-Lachapelle A. Jour de fête! BioéthiqueOnline. 2016;5/17.

- Ménard J-F. Cobayes de la relève : l'artiste et le chercheur à la rencontre de l'expérience. BioéthiqueOnline. 2016;5/18.

- Coleman S. Na-no-body. BioéthiqueOnline. 2016;5/19.

- Noury M. Na-no-body: De l'oubli du corps sensible en nanomédecine. BioéthiqueOnline. 2016;5/20.

- Stokes G. Constant Beauty Within and PCOS as Cacti. BioéthiqueOnline. 2016;5/21.

- Doudenkova V. La bioéthique, l'art et le syndrome des ovaires polykystiques: propos impressionniste visant à réhabiliter les corps tabous et les ovaires blâmés. BioéthiqueOnline. 2016;5/22.

- Kinkead A. 54 rue DuBalcon. BioéthiqueOnline. 2016;5/23.

- Rathwell-Deault D. L'animal un co-citoyen, et pourquoi pas? BioéthiqueOnline. 2016;5/24.

- Turcot K. «Médecine à deux vitesses à la manière de SUCCESs » ou « Deux poids deux mesures ou Quod licet lovi, non licet bovi c'est-à-dire "ce qui est permis à Jupiter ne l'est pas aux vaches" „) BioéthiqueOnline. 2016;5/25.

- Carrier A, Contandriopoulos D. Principes de communication et rôle social du chercheur en matière de transfert de connaissances: une dualité source de questionnements éthiques. BioéthiqueOnline. 2016;5/26.

- Le Mée M. Mael-e(s)t-France. BioéthiqueOnline. 2016;5/27.

- Beaudry J-S. The anxious heart of injustice: negative affective responses to disabilities. BioéthiqueOnline. 2016;5/28.

- Barnabé C. Art + Bioéthique : L'art comme médiation. BioéthiqueOnline. 2016;5/29.

- Cloutier M. Faire dialoguer les cultures : rencontre entre la bioéthique et l'art contemporain. BioéthiqueOnline. 2016;5/30.

- Lorrain A. Art + Bioéthique : Exposition et évènements sous le signe de la collaboration entre l'éthique et l'esthétique. BioéthiqueOnline. 2016;5/31. 